\title{
Norois
}

Environnement, aménagement, société

202 | 2007/1

Recomposition des espaces ruraux

\section{Quel risque de pénurie d'eau sur les îles du Ponant?}

Which water shortage risk for the Ponant Islands?

\section{Thomas Chiron}

\section{(2) OpenEdition}

Journals

Édition électronique

URL : https://journals.openedition.org/norois/1644

DOI : 10.4000 /norois. 1644

ISBN : 978-2-7535-1550-5

ISSN : 1760-8546

\section{Éditeur}

Presses universitaires de Rennes

Édition imprimée

Date de publication : 1 mars 2007

Pagination : 73-86

ISBN : 978-2-7535-0457-8

ISSN : 0029-182X

\section{Référence électronique}

Thomas Chiron, «Quel risque de pénurie d'eau sur les îles du Ponant? », Norois [En ligne], 202 | 2007/1,

mis en ligne le 01 mars 2009, consulté le 14 janvier 2022. URL : http://journals.openedition.org/norois/ 1644 ; DOI : https://doi.org/10.4000/norois. 1644 


\title{
Quel risque de Pénurie d’eau sur les îles du Ponant?
}

\author{
ThOMAS CHIRON \\ GÉOMER - LETG UMR 6554 CNRS \\ (Université de Bretagne Occidentale), \\ Institut Universitaire Européen de la Mer - Technopôle Brest-Iroise, \\ place Nicolas-Copernic - 29285 PlouZAné \\ thomas.chiron@univ-brest.fr
}

\begin{abstract}
RÉSUMÉ
les îles du Ponant constituent un groupe de quinze petites îles côtières françaises, en Atlantique et en Manche. Soumises au climat tempéré océanique, elles connaissent des conditions hydroclimatiques marquées par la variabilité interannuelle de leurs bilans hydriques. Aussi la recharge de leurs réserves hydriques, par ailleurs limitées, est-elle aléatoire: le risque de pénurie est donc étudié du point de vue climatique pour les îles ayant conservé leur insularité hydraulique. Les cas de Belle-Île et Molène sont développés selon des scenarios prospectifs intégrant différents facteurs, sociaux et physiques.
\end{abstract}

Mots CLÉ : Aléa climatique - Îles - Pénurie d'eau-Ressources en eau-Risque.

\section{ABSTRACT}

\section{Which water shortage risk for the Ponant Islands?}

this contribution is focused on the Ponant Islands which are a group of fifteen small islands off the Western French coast and where fresh water question is crucial because of restricted resources. The research aims at assessing island fresh water shortage risk due to unfavourable hydroclimatic, structural conditions and anthropic pressure on water resources. Two case studies are developed thanks to prospective scenarios on water demand and infrastructures: Belle-Île and Molène.

KEY WORDS : Climatic Factor - Islands - Risk - Water Resources - Water Shortage.

La littérature a souvent fait état du problème de l'eau potable sur les îles du Ponant, sans autre développement exhaustif sur la question. Elle s'est contentée de souligner les dangers induits par les modèles de consommation contemporains (Bioret et al., 1991) qui, en contexte insulaire, menacent les équilibres environnementaux tant le caractère fini des ressources y est perceptible (Singelin et Monnat, 1991 ; Péron, 1993; Brigand, 1999). Ainsi, dans les années 1960-1970, « [...] le manque d'eau, lié à l'insularité, [a été] cruellement ressenti dans toutes les îles de l'Atlantique, d'autant plus que le développement touristique amène un gonflement énorme de la population pendant les mois d'été » (Dumortier, 1976). Les îles du Ponant dans leur ensemble ont effectivement connu, depuis les années 1950 et surtout le début des années 1960, de profondes mutations socioéconomiques : elles sont passées d'îles à activités traditionnelles - pêche et agriculture, 
au statut d'« îles consommées » sous l'influence de leur attractivité touristique. Conjuguée à cette dernière, l'amélioration générale du confort domestique dans les années 1960 et 1970 (Guilcher, 1977 ; Brigand, 1983) a augmenté les besoins en eau alors que les ressources des îles semblent $a$ priori limitées physiquement. De plus, la saisonnalité de la fréquentation touristique concentre la demande durant la saison estivale qui est précisément une saison de déficit hydrique (Chiron et Dubreuil, 2006) : les îles doivent donc disposer de réserves hydriques suffisantes pour satisfaire cette demande.

La pénurie d'eau résulte justement d'une insuffisance quantitative et/ou qualitative de la ressource hydrique disponible par rapport à la demande. De nombreuses recherches interdisciplinaires sont menées sur cette problématique essentielle du développement durable des sociétés, participant ainsi à la réflexion pour une meilleure répartition et préservation de l'eau (Rivière-Honegger et Bravard, 2005). Deux indicateurs sont couramment utilisés pour caractériser la pénurie d'eau (Margat, 2005) :

- l'abondance ou la rareté relative des ressources en eau, qui compare les ressources disponibles (en terme de flux moyen) à la population considérée a priori comme le principal facteur universel de demande en eau,

- la pression humaine sur les ressources en eau, qui compare les prélèvements aux flux moyens de ressources.

Ces indicateurs posent pourtant deux problèmes majeurs : d'une part, celui de la référence territoriale qui, souvent considérée à l'échelle régionale ou nationale, masque des disparités locales ; d'autre part, celui de la définition même des ressources en eau, laquelle se réfère généralement aux seules ressources renouvelables naturelles alors qu'il serait plus réaliste de considérer l'ensemble des ressources exploitables.

La question de la pénurie d'eau sur les îles du Ponant fait écho à ces préoccupations méthodologiques fondamentales en focalisant la réflexion sur des territoires exigus et isolés. Elle y est d'autant plus importante que les enjeux socioéconomiques liés au tourisme sont directement concernés par la limite physique naturelle imposée par les ressources endogènes, l'eau tout particulièrement. Les histoires insulaires ont d'ailleurs été marquées par de vraies crises de l'eau; récemment, les difficultés de sécurisation de l'approvisionnement en eau potable de Belle-Île durant le second semestre 2005 ne manquent pas de rappeler combien la problématique reste sensible pour certaines de ces petites îles. Il s'agit donc de répondre à la question du risque de pénurie d'eau en apportant des développements méthodologiques et des résultats objectifs nouveaux adaptés au contexte géographique local et fondés sur deux indicateurs. La vulnérabilité hydraulique des îles tient ici exclusivement aux facteurs sociaux (besoins en eau potable); l'aléa n'est quant à lui considéré que du seul point de vue climatique. Aussi le contexte hydroclimatologique des îles du Ponant est-il préalablement rappelé. Le risque de pénurie est ensuite défini et quantifié, l'analyse choisie permettant des comparaisons spatio-temporelles. Enfin, l'étude de deux exemples, Belle-Île et Molène, propose des visions rétrospectives et prospectives sur la gestion du risque de pénurie sur deux îles très différentes.

\section{Hydromorphologie et hydroclimatologie des îles du Ponant}

\section{DES RESSOURCES HYDRIQUES PHYSIQUEMENT LIMITÉES}

Les îles du Ponant désignent un groupe de quinze petites îles françaises disséminées sur les côtes métropolitaines de l'Atlantique et de la Manche. A l'exception de l'île d'Aix, plus méridionale et située en marge du bassin sédimentaire aquitain, ces îles jalonnent le pourtour des côtes bretonnes, se situant ainsi en périphérie du Massif armoricain. Elles sont caractérisées par la diversité de leurs situations géographiques (fig. 1) : les îles à caractère continental peuvent être distinguées d'îles plus petites, soit proches du continent soit plus au large et donc plus océaniques. Elles présentent une géologie générale de socle caractérisée par la prédominance des roches granitiques 


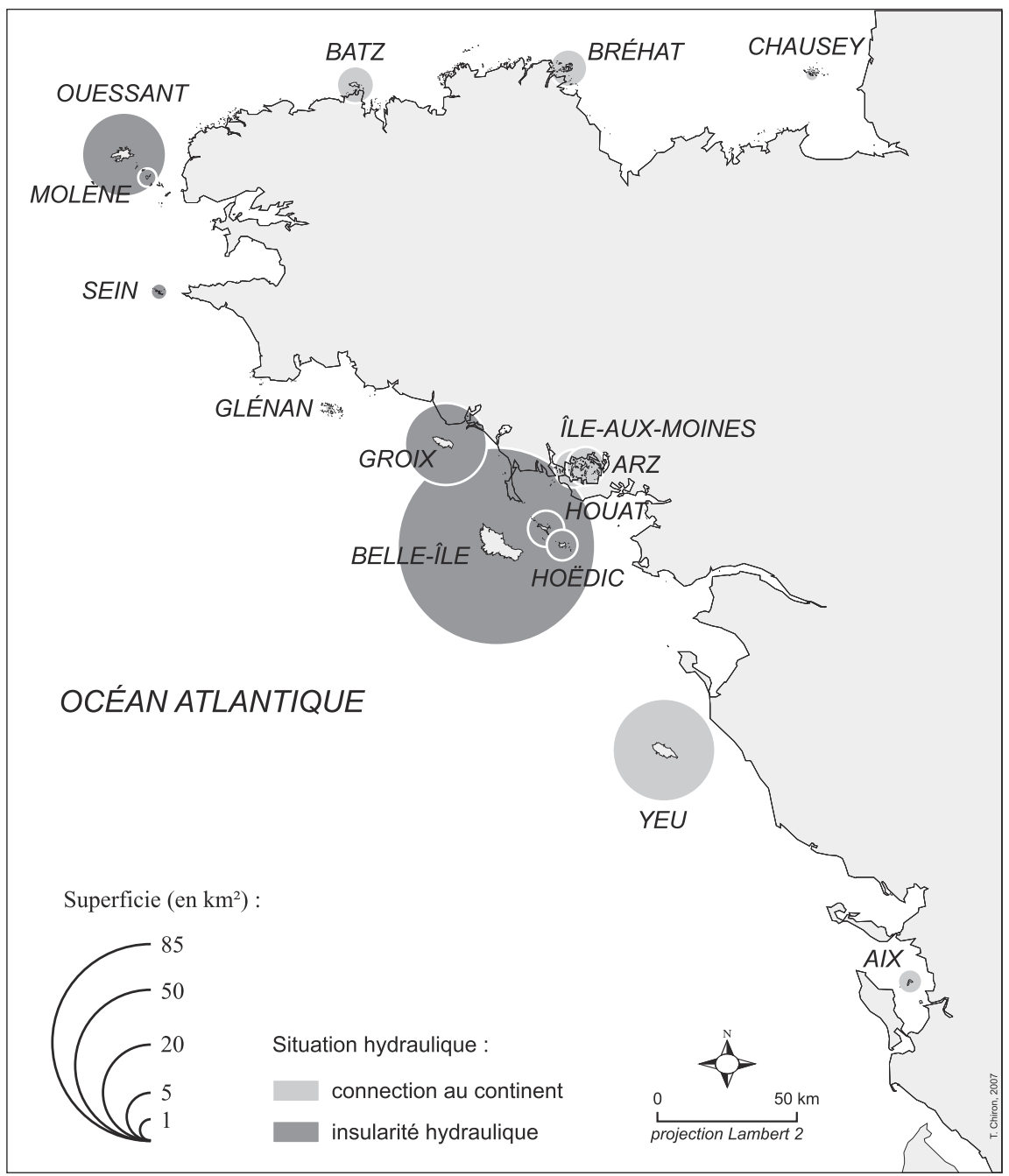

Figure 1 : Les Îles du Ponant : superficie et situation hydraulique Area and hydraulic condition of the Ponant Islands

et schisteuses dont le potentiel capacitif se limite aux fissures et formations arénisées, voire aux accumulations sédimentaires en coiffe de socle. Du point de vue hydrogéologique, « [...] les nappes profondes sont donc peu fournies et spatialement peu étendues. Ceci signifie que les déficits pluviométriques se traduisent rapidement par une chute des débits hydrologiques » en l'absence de soutien des étiages (Dubreuil, 1994). Sur les quatre plus grandes îles - Belle-Île $\left(85,6 \mathrm{~km}^{2}\right)$, Yeu $\left(23,3 \mathrm{~km}^{2}\right)$, Ouessant $\left(15,6 \mathrm{~km}^{2}\right)$ et Groix $\left(14,8 \mathrm{~km}^{2}\right)$, les réseaux hydrographiques sont peu développés du fait de bassins versants topographiques restreints à quelques dizaines d'hectares. Ils restent embryonnaires sur les plus petites, à l'instar de Houat $\left(3 \mathrm{~km}^{2}\right)$, où ils sont circonscrits à de petits vallons côtiers de quelques dizaines d'ares, voire quasi inexistants à Molène $\left(0,75 \mathrm{~km}^{2}\right)$ et Sein $\left(0,6 \mathrm{~km}^{2}\right)$. Il n'existe aucun cours d'eau permanent sur l'ensemble des quinze îles : durant la saison sèche, les rares ruisseaux qui coulent en hiver et au printemps s'assèchent irrémédiablement. De nombreuses résurgences et sources sont pourtant recensées sur les littoraux insulaires, la présence de fontaines, de lavoirs et de puits sur l'ensemble des îles attestant de l'utilisation 
historique de cette eau souterraine. L'exiguïté spatiale insulaire rend cependant doublement délicate l'exploitation contemporaine de ces ressources hydrogéologiques : d'une part les volumes mobilisables sont limités physiquement, d'autre part la proximité de la mer les rend vulnérables aux intrusions salines en cas de surexploitation. Les ressources en eau sont donc a priori physiquement limitées sur l'ensemble des îles du Ponant, avec toutefois un potentiel hydrologique plus intéressant pour les îles plus grandes.

\section{NORMALITÉ ET VARIABILITÉ INTERANNUELLE DES BILANS HYDRIQUES}

Les îles du Ponant sont situées dans le domaine climatique tempéré océanique. Plus précisément, elles sont soumises au climat breton qui caractérise la marge littorale depuis le Jutland au Danemark jusqu'aux provinces cantabriques d'Espagne (Viers et Vigneau, 1990). Une première originalité des côtes bretonnes réside dans l'absence de reliefs insulaires et littoraux marqués, laquelle engendre la dominance des pluies frontales par opposition aux pluies orographiques. La fréquence des précipitations est élevée mais les totaux pluviométriques sont modestes, ce qui traduit de faibles intensités moyennes. Calculées sur la période 1949-2005, les précipitations annuelles moyennes des îles du Ponant ne dépassent pas $850 \mathrm{~mm}$ : un maximum de $832 \mathrm{~mm}$ à Batz, $708 \mathrm{~mm}$ à Ouessant et $680 \mathrm{~mm}$ à Belle-Île. De plus, l'inertie thermique de l'océan et sa fraîcheur pendant l'été atténuent l'instabilité, ce qui a pour conséquence une diminution de la pluviométrie estivale par rapport aux pluies hivernales. Les mois de juin, juillet et août sont en moyenne les moins arrosés. Cette déficience pluviométrique estivale se traduit par une sécheresse météorologique relative qui, avec le domaine méditerranéen, reste une exception climatique à l'échelle du globe (Viers et Vigneau, 1990).

Dressés à partir du calcul de l'évapotranspiration selon la formule de Penman, les bilans hydriques mensuels de neuf stations des îles du Ponant - Grande-Île, Bréhat, Batz, Ouessant, Sein, Groix, Belle-Île, Houat et Aix - montrent que la part moyenne annuelle des précipitations dédiée à la recharge des réserves hydriques par ruissellement et infiltration - les pluies efficaces selon l'acception de G. Castany (1982) - est comprise entre $25 \%$ et $34 \%$. Celle-ci se concentre quasi exclusivement au cours du premier semestre de l'année hydrologique : d'octobre à mars. Généralement réamorcées en octobre, les pluies efficaces engendrent une nette augmentation de la part du ruissellement et de l'infiltration dès le mois de novembre pour atteindre des maxima en décembre, janvier et février. Ces trois mois concentrent en moyenne les deux tiers voire les trois quarts des surplus hydrologiques annuels selon les îles, et plus de $95 \%$ des pluies efficaces sont enregistrées au cours du premier semestre hydrologique. En moyenne, entre $180 \mathrm{~mm}$ et $270 \mathrm{~mm}$ sont mobilisés pour la recharge des réserves hydrologiques (Chiron et Dubreuil, 2006).

J. Mounier (1980) rappelle cependant qu'il est nécessaire de prendre en compte la variabilité interannuelle des éléments du climat pour mieux comprendre la contrainte climatique qui s'exerce sur un territoire, sa population et ses activités économiques. La variabilité interannuelle est justement caractéristique des bilans hydriques des îles du Ponant. Ces derniers peuvent être perturbés par un manque de précipitations hivernales et/ou des conditions de température favorisant une évapotranspiration très forte au printemps et en été. La vulnérabilité hydrique des îles se trouve principalement assujettie à ces perturbations du cycle de l'eau. Les bilans hydriques montrent que le cumul annuel des pluies efficaces est variable d'une année sur l'autre. Le tableau 1 recense les années remarquables pour lesquelles les pluies efficaces n'ont pas atteint $25 \%$ des valeurs moyennes calculées. Certaines années sont même marquées par l'absence de reprise du ruissellement et de l'infiltration. Les réserves hydriques superficielles et souterraines insulaires ne sont alors pas ou que partiellement rechargées : la pénurie est à craindre si le stock d'eau disponible ne couvre pas les besoins en eau potable de l'année hydrologique en cours. Ce sera le cas lors des sécheresses historiques de 1989 - où les îles de Molène, Groix, Belle-Île, Houat et Hoëdic seront ravitaillées en eau par bateau, et de 2005 à Belle-Île (Chiron et Dubreuil, 2006). 


\begin{tabular}{|c|c|c|c|c|}
\hline Île & Période & $P_{\text {eff }} \leq 25 \% \overline{P_{\text {eff }}}$ & $P_{\text {eff }} \leq 10 \% \overline{P_{\text {eff }}}$ & $P_{\text {eff }}=0$ \\
\hline Bréhat & $1949-2005$ & 1962 & $1954,1976,1989$ & 1963,2002 \\
\hline Batz & $1949-2005$ & 1987 & 1976 & $1954,1989,1992$ \\
\hline Ouessant/Molène & $1949-2005$ & 1967,1976 & 1954,2005 & 1989,1992 \\
\hline Sein & $1974-2005$ & 1997 & & $1989,1992,2002,2005$ \\
\hline Groix & $1949-2005$ & 1954 & $1987,1989,2002,2005$ \\
\hline Belle-Île & $1949-2005$ & 1949,2002 & 1989,2005 \\
\hline Houat/Hoëdic & $1966-2005$ & $1976,1989,2002$ & & 1967 \\
\hline Aix & $1979-2005$ & 2005 & & 2002 \\
\hline
\end{tabular}

Tableau 1 : Classification des années hydrologiques à faible recharge hydrique en fonction des pourcentages de pluies efficaces calculées $\left(P_{\text {eff }}\right)$ par rapport à la moyenne $\left(\bar{P}_{\text {eff }}\right)$.

Classification of years with low hydrous refill, according to calculated efficient rain $\left(P_{\text {eff }}\right)$ in com-
parison with average value $\left(\boldsymbol{P}_{\text {eff }}\right)$.

La saison sèche qui caractérise les étés armoricains succède donc à une période assez courte de remplissage automnal et hivernal des réserves hydriques insulaires. Les facteurs naturels - hydromorphologiques et hydroclimatiques - corroborent donc a priori la limitation physique de l'offre hydrique des îles relativement à la demande anthropique.

\section{Quel risque de pénurie d'eau sur les îles du Ponant?}

\section{L'INSULARITÉ HYDRAULIQUE}

La saisonnalité et la variabilité interannuelle des conditions hydroclimatiques des îles du Ponant sont des caractéristiques majeures de la problématique de la gestion de leurs ressources en eau. L'enjeu posé est celui de la sécurité de l'approvisionnement en eau potable des populations insulaires à partir des ressources hydriques endogènes. Les îles ont connu une érosion démographique de près de $50 \%$ de leurs populations permanentes au cours du XXe siècle : 29369 habitants étaient recensés en 1911 contre 15223 en $1999^{1}$; paradoxalement, une forte augmentation des besoins en eau est constatée dès l'après-guerre sous l'influence de l'amélioration générale du niveau de vie et de la croissance de la fréquentation touristique. Dans les années 1960 et au début des années 1970, alors qu'apparaissent les premières difficultés liées à un déficit hydrique d'origine structurelle, « la solution la plus habituelle est le raccordement aux ressources du continent par une conduite sous-marine » (Patturel, 1972). Six îles ont ainsi été connectées aux réseaux continentaux d'adduction d'eau potable : Bréhat, Batz, l'Île-aux-Moines, Arz, Yeu et Aix. En 1972, le directeur de l'Aménagement rural et des structures au ministère de l'Agriculture, M. Blaizot, précise pourtant que « lorsque les îles à desservir sont de faible superficie et, par conséquent, les volumes d'eau à importer limités, [et] lorsque les distances à partir du rivage sont importantes, les fonds intermédiaires rocheux et les mouvements de la mer violents, la solution de la conduite sous-marine est techniquement difficile et économiquement prohibitive » (Patturel, 1972).

Parmi les neuf îles non raccordées au continent, les îles de Grande-Île dans l'archipel de Chausey et de Saint-Nicolas-des-Glénan sont régulièrement ravitaillées en eau douce par bateau par les communes littorales dont elles dépendent administrativement. L'île de Sein s'est quant à elle affranchie dès 1976 de l'absence de ressource hydrique en produisant son eau potable par désalinisation de l'eau de mer. En somme, seules six îles ont conservé une insularité hydraulique strictement fondée sur l'exploitation de ressources hydriques conventionnelles endogènes pour satisfaire les besoins en eau potable de leurs populations permanentes et touristiques : Ouessant 

(335 hab.) et Hoëdic (117 hab.) ${ }^{2}$.

\section{UN INDICATEUR DE VULNÉRABILITÉ : L’AUTONOMIE HYDRAULIQUE}

Défini comme le produit de l'aléa et de la vulnérabilité (Dauphiné, 2003), une meilleure connaissance du risque de pénurie d'eau d'origine climatique constitue un atout indéniable dans la maîtrise et l'exploitation rationnelle et durable des ressources en eau des îles en situation d'autonomie hydraulique. L'aléa est ici climatique et la vulnérabilité ne tient finalement qu'au seul facteur humain qui définit la pression exercée sur les ressources hydriques disponibles. Leur évaluation est fondée sur le diagnostic des volumes de stockage (lacs de retenue et citernes) et l'estimation du volume exploitable des aquifères insulaires selon les capacités nominales de pompage ou les recommandations techniques d'exploitation. Les volumes des réserves hydriques souterraines sont directement issus des rapports de la Direction Départementale de l'Agriculture et de la Forêt du Morbihan (1991) pour Hoëdic, des cabinets de consultants Sogreah-Praud (2004) pour Houat et Antea pour Molène (Anonyme, 2001), et des données communiquées par la société Véolia-Eau en tant que gestionnaire délégataire du service d'eau pour Groix (communication personnelle).

Le degré d'autonomie hydraulique de l'île est alors défini comme le ratio entre le volume des besoins anthropiques en eau et celui des ressources hydriques disponibles. En agrégeant des données tant physiques que sociales, l'autonomie hydraulique insulaire est un indicateur simple mais pertinent pour mesurer la vulnérabilité des îles : elle traduit la durée pendant laquelle elles peuvent vivre sur leurs propres réserves en eau sans que celles-ci ne soient réalimentées. Les îles dont l'autonomie hydraulique est inférieure à deux ans restent très vulnérables à l'aléa sécheresse : l'insuffisance ou pire l'absence de reconstitution de leur réserve au cours de l'automne et de l'hiver risquent d'engendrer une rupture de l'alimentation en eau potable des populations îliennes au cours de l'été ou au début de l'automne suivant. En se référant aux données de l'année 2005, les situations d'autonomie hydraulique des six îles sont hétérogènes (tableau 2). Les îles de Houat et Hoëdic, où seules les ressources souterraines sont exploitées, présentent des autonomies hydrauliques supérieures à deux ans (respectivement 2,3 et 2,8 ans); celle de l'île de Groix, qui dispose de ressources d'origine mixte - superficielle et souterraine - est également légèrement supérieure à deux ans $(2,2)$. Pourtant, une augmentation des besoins de $11 \%$ la replacerait dans une situation d'autonomie hydraulique inférieure au seuil critique de deux ans. C'est la situation que connaissent les trois autres îles : Belle-Île et Molène présentent une autonomie d'une année et demi environ. La situation de l'île d'Ouessant est plus délicate encore puisque les besoins annuels sont plus importants que la capacité de stockage de l'île : son autonomie hydraulique n'est ainsi que de 0,8 an. En cas de déficit pluviométrique hivernal très prononcé, les réserves de ces trois îles risquent de ne pas se recharger suffisamment pour suffire aux besoins de l'été suivant.

\section{Proposition de QUANTIFICATION DU RISQUe DE PÉNURIE D’EAU}

L'aléa est un concept statistique : la fréquence au dépassement ou au non-dépassement d'un événement climatique constitue un indicateur particulièrement bien adapté à l'appréciation du problème posé. Il s'agit ici d'estimer la fréquence au non-dépassement des cumuls de précipitations mensuelles du premier semestre hydrologique pour lesquels la recharge des réserves hydriques ne suffirait pas à satisfaire les besoins annuels en eau potable. Principal paramètre climatique utilisé en hydroclimatologie, les hauteurs de précipitations se prêtent classiquement à une distribution selon une loi mathématique. Un tel ajustement est un préalable nécessaire à la signification géographique de l'analyse statistique menée : la distribution lui offre un sens mathématique et peut alors être utilisée comme un intégrateur de différentes tendances permettant des comparaisons 
QUEL RISQUE DE PÉNURIE D’EAU SUR LES ÎLES DU PONANT?

\begin{tabular}{|l|c|c|c|c|c|}
\hline \multirow{2}{*}{ Îles } & $\begin{array}{c}\text { Besoins annuels } \\
\left(\mathrm{m}^{3}\right)\end{array}$ & \multicolumn{3}{|c|}{ Volume des réserves hydriques $\left(\mathrm{m}^{3}\right)$} & $\begin{array}{c}\text { Autonomie } \\
\text { hydraulique } \\
\text { (années })\end{array}$ \\
\cline { 2 - 5 } & 20000 & stockage & souterraines & totales \\
\hline Hoëdic & 24000 & 12100 & 50000 & 57600 & 2,8 \\
Houat & 140000 & 160000 & 150000 & 55100 & 2,3 \\
Groix & 6500 & 1850 & 10000 & 118000 & 2,2 \\
Molène & 600000 & 850000 & - & 850000 & 1,8 \\
Belle-Île & 70000 & 55000 & - & 55000 & 1,4 \\
Ouessant & \multicolumn{2}{|l}{} &
\end{tabular}

Tableau 2 : Autonomie hydraulique des îles, situation référence de 2005 Island hydrological range, reference year: 2005

spatio-temporelles (Marchand, 1981). Les distributions des précipitations mensuelles et annuelles ont été modélisées depuis de nombreuses années par les travaux d’EDF qui utilisent la loi Gamma incomplète (Péguy et al., 1981 ; Marchand, 1981).

L'analyse statistique a été menée sur les cumuls des hauteurs de précipitations mensuelles du premier semestre hydrologique enregistrées de 1949 à 2005 - sauf pour l'île de Houat où les données météorologiques ne sont disponibles qu'à partir de 1966. Les résultats pour les stations d'Ouessant et Houat ont été respectivement transposés aux îles de Molène et Hoëdic compte tenu de leur proximité. Les ajustements des échantillons ont été calculés avec les logiciels EXCEL® et STATISTICA $®$ : ils sont validés a posteriori à l'aide du test de Kolmogorov-Smirnov. Ce premier traitement permet de connaître la fréquence d'occurrence des cumuls de précipitations au premier semestre hydrologique.

Les pluies efficaces conditionnant la recharge des réserves hydriques - le volume de recharge correspond au produit de la surface du bassin versant et de la hauteur de pluies efficaces, une relation entre les cumuls de hauteurs mensuelles de précipitations et les pluies efficaces mensuelles du premier semestre hydrologique est ensuite établie. Ils sont corrélés linéairement, avec des coefficients de corrélation $\mathrm{R}^{2}$ proches de 0,9 (tableau 3). Connaissant les surfaces réceptrices sur chacune des îles, il est facile de déterminer la valeur seuil des pluies efficaces nécessaires à la recharge minimale souhaitée : en première approximation, celle-ci sera égale aux besoins annuels en eau. Rétroactivement, selon la relation linéaire établie précédemment, cette valeur seuil de pluies efficaces est corrélée à un cumul de précipitations au premier semestre hydrologique dont la fréquence d'occurrence est connue. Le risque de non satisfaction des besoins est ainsi quantifié par cette fréquence selon une démarche qui intègre à la fois les facteurs naturels et sociaux.

\begin{tabular}{|l|c|c|}
\hline Îles & Relation & $\mathrm{R}^{2}$ \\
\hline Ouessant/Molène & $\mathrm{P}_{\text {eff }}=1 * \mathrm{P}-280$ & 0,92 \\
Groix & $\mathrm{P}_{\text {eff }}=0,8 * \mathrm{P}-226$ & 0,89 \\
Belle-Île & $\mathrm{P}_{\text {eff }}=0,9 * \mathrm{P}-188$ & 0,89 \\
Houat/Hoëdic & $\mathrm{P}_{\text {eff }}=0,9 * \mathrm{P}-194$ & 0,93 \\
\hline
\end{tabular}

Tableau 3 : Relation linéaire entre pluies efficaces $\left(\mathrm{P}_{\text {eff }}\right)$ et précipitations $(\mathrm{P})$ du premier semestre hydrologique (Période 1966-2005, en millimètres)

Linear relationship between efficient rainfall and rainfall during the first hydrological semester (Period 1966-2005, in millimeters) 
Le tableau des résultats pour la situation référence de 2005 (tableau 4) corrobore la dichotomie des situations insulaires face à ce risque de pénurie : d'une part les îles du département du Morbihan - Houat, Hoëdic et Groix - et d'autre part Belle-Île et les îles finistériennes d'Ouessant et de Molène. Sur les premières, le risque que les pluies efficaces du premier semestre ne suffisent à produire une recharge hydrique égale aux besoins annuels est estimé avec une fréquence d'une année sur vingt (Groix) voire sur trente (Houat et Hoëdic). De plus, leur autonomie hydraulique leur garantit une sécurité de l'approvisionnement en eau potable face à de tels aléas pluviométriques. À la suite de la pénurie de 1989, des recherches d'eau souterraine ont été menées sur ces trois îles : elles ont conduit à la mise en place de forages qui ont permis d'augmenter sensiblement les réserves hydriques exploitables et les surfaces réceptrices. Leur vulnérabilité hydraulique a diminué, tout comme le risque de pénurie, puisque les volumes à mobiliser le sont avec des pluies efficaces - et donc des précipitations - moins importantes : la fréquence au non-dépassement des seuils pluviométriques requis est par conséquent moins grande. En revanche, pour les trois autres îles, le risque est d'autant plus élevé qu'elles sont plus vulnérables hydrauliquement. Ainsi, Belle-Île et Ouessant risquent de connaître des difficultés d'approvisionnement en eau potable une année sur treize et quinze ; cette fréquence tombe même à une année sur neuf à Molène.

\begin{tabular}{|l|c|c|c|c|c|}
\hline Îles & $\begin{array}{c}\text { Besoins annuels } \\
\left(\mathrm{m}^{3}\right)\end{array}$ & $\begin{array}{c}\text { Superficie des } \\
\text { bassins versants } \\
\left(\mathrm{km}^{2}\right)\end{array}$ & $\begin{array}{c}\text { Seuil de pluies } \\
\text { efficaces } \\
(\mathrm{mm})\end{array}$ & $\begin{array}{c}\text { Hauteurs de } \\
\text { précipitations } \\
\text { corrélées }(\mathrm{mm})\end{array}$ & $\begin{array}{c}\text { Fréquence } \\
\text { d'occurrence }\end{array}$ \\
\hline Houat & 24000 & 1 & 16 & 231 & $1 / 30$ \\
Hoëdic & 20000 & 1 & 20 & 235 & $1 / 30$ \\
Groix & 140000 & 10 & 14 & 268 & $1 / 20$ \\
Belle-île & 600000 & 10 & 30 & 227 & $1 / 13$ \\
Ouessant & 70000 & 1,5 & 47 & 304 & $1 / 15$ \\
Molène & 6500 & 0,1 & 65 & 324 & $1 / 9$ \\
\hline
\end{tabular}

Tableau 4 : Estimation du risque de non-satisfaction interannuelle des besoins, situation référence de 2005 Assessment of annual water shortage risk, reference year: 2005

Le risque de pénurie tel qu'il est défini peut évoluer en fonction de deux facteurs anthropiques, liés au développement socioéconomique insulaire :

- les besoins de la population insulaire, permanente et saisonnière, ble.

- la mobilisation de la ressource : les équipements de production et de stockage d'eau pota-

Il est à ce titre intéressant de s'attarder sur les cas de Belle-île et Molène. L'exemple ouessantin n'est pas développé malgré sa faible autonomie hydraulique : sa situation est assez similaire à celle de Belle-Île à ceci près qu'elle présente une constance relative des volumes d'eau distribués depuis une douzaine d'années et qu'elle n'a pas connu de pénurie d'eau depuis 1972. Finalement, quatre raisons justifient le choix de ces deux îles:

- elles présentent un risque de pénurie non négligeable,

- elles sont géographiquement très différentes (taille et population),

- elles ont connu de graves épisodes de pénuries d'eau, notamment en 1989 où elles ont été ravitaillées par bateau (tout comme Houat, Hoëdic et Groix),

- en se fondant sur les tendances observées et leurs perspectives de développement urbanistique, elles devraient être soumises à court et moyen terme à une augmentation sensible des besoins en eau. 


\title{
Évolution rétrospective et prospective du risque de pénurie d'eau sur les îles de Belle-île et Molène
}

\author{
CONSTRUCTION DE SCÉNARIOS EXPLORATOIRES TENDANCIELS
}

Les développements méthodologiques précédents invitent à s'intéresser aux évolutions possibles du risque de pénurie à moyen terme, dans la perspective d'une aide à la prise de décision dans les politiques de gestion locale des ressources en eau insulaires. L'effort de prospective peut s'appuyer sur la méthodologie des scénarios, lesquels peuvent être définis comme "des séquences d'événements hypothétiques construites pour mettre en évidence les processus causaux et les enjeux de décision » (Kahn et Wiener, 1968). Il s'agit de développer des outils qui permettent d'appréhender les dynamiques futures d'objets ou de systèmes complexes, par nature indéterminés, conduisant à une pluralité de futurs possibles. Plusieurs types de scénarios sont envisageables, parmi lesquels les scénarios exploratoires : le présent est alors la situation de référence à partir de laquelle des projections sont envisagées en fonction d'hypothèses sur les variables d'évolution en jeu. Les scénarios tendanciels, qui sont une déclinaison des précédents, envisagent plus précisément les conséquences de la continuation des tendances de la situation de référence, en conservant les dynamiques en cours (Poux, 2005).

Dans le contexte insulaire considéré, où il n'y a pas d'agriculture irriguée ni d'industries grandes consommatrices d'eau, les dynamiques en cours sont liées aux seuls besoins domestiques. Les scénarios tendanciels envisagés sont construits à partir de deux hypothèses $\mathrm{H} 1$ et $\mathrm{H} 2$ :

- (H1) : l'évolution interannuelle des besoins est modélisée par une croissance linéaire de la demande domestique en eau, d'après les droites de corrélation sur les besoins en eau des vingt (Molène) ou trente (Belle-Île) dernières années.

- (H2) : les perspectives urbanistiques des deux îles à court (2010) et moyen terme (2020) conditionnent l'augmentation des besoins en eau; la consommation annuelle de chaque nouvelle résidence est estimée à $100 \mathrm{~m}^{3}$, conformément à la consommation moyenne d'un ménage français de quatre personnes.

Pour la seconde hypothèse $\mathrm{H} 2$, les développements urbanistiques de Belle-Île et de Molène sont déterminés selon des projections sur le rythme de construction résidentielle, projections fondées sur les données INSEE, les Plans Locaux d'Urbanisme (PLU) et les conclusions de la thèse de C. Buhot (2006) sur le marché immobilier insulaire. Pour Belle-Île, le rythme actuel de construction se situe autour d'une centaine de nouvelles résidences par an. D’après C. Buhot (2006), leur nombre est limité à cinq cents, ce qui pourrait stopper le développement urbanistique de l'île en 2010. Le scénario sera cependant prolongé à l'horizon 2020. Validé en 2006, le PLU de l'île de Molène prévoit quant à lui la construction possible de trente-quatre résidences. Le rythme actuel se situant autour de deux nouvelles constructions par an, il peut être admis qu'une dizaine sera construite entre 2005 et 2010 , et que l'ensemble pourrait être achevé en 2020. Une variante de la seconde hypothèse ( $\mathrm{H} 2$ bis) est également déclinée compte tenu du contexte molénais : elle intègre l'équipement des nouvelles résidence en citernes de récupération des eaux de pluie. Ce paramètre est fondamental dans la problématique locale de gestion de l'eau puisque la récupération des eaux de pluie est une pratique traditionnelle sur l'île : elle permet actuellement de maintenir la demande en eau du service public à une moyenne de $30 \mathrm{~m}^{3}$ par résidence, résidences principales et secondaires confondues.

\section{VERS UNE PRÉCARISATION HYDRAULIQUE À BELLE-ÎLE?}

La sécheresse de 2005 a révélé la vulnérabilité hydraulique de Belle-Île : l'île a dû être ravitaillée par bateau cargo pour éviter une pénurie au cours du second semestre 2005 et au premier semestre 2006. Lîle avait connu une situation similaire pendant la sécheresse de 1989 : il avait alors été décidé de construire une troisième retenue d'eaux superficielles, augmentant ainsi les réserves 
hydriques de $330000 \mathrm{~m}^{3}$ à $850000 \mathrm{~m}^{3}$. La vulnérabilité hydraulique de l'île a de la sorte baissé entre 1989 et 2005 ; paradoxalement, l'analyse rétrospective de l'évolution du risque de pénurie d'eau montre que celui-ci n'a pas diminué. L'augmentation des surfaces pourvoyeuses d'eau de 7 à $10 \mathrm{~km}^{2}$ suffit juste à compenser celle des besoins qui n’ont cessé de croître au cours des années 1990, passant de $440000 \mathrm{~m}^{3}$ en 1989 à $600000 \mathrm{~m}^{3}$ en 2005 : les pluies efficaces minimales nécessaires à la mobilisation des volumes requis pour satisfaire les besoins sont sensiblement égales. En réponse à la crise hydrique de 2005, l'installation de stations de pompage des eaux de ruissellement dans des bassins versants supplémentaires a permis d'augmenter les surfaces réceptrices de $10 \mathrm{~km}^{2}$ à près de $24 \mathrm{~km}^{2}$ au début de l'année 2006. Bien que la réserve hydrique se maintienne à $850000 \mathrm{~m}^{3}$, cela permet théoriquement de mobiliser les volumes requis même au cours d'années peu arrosées : de 2005 à 2006, l'accroissement du potentiel hydrique de l'île augmente ainsi la fréquence d'occurrence de l'événement critique d'une année sur treize à une année sur trente-cinq (tableau 5).

Les scénarios prospectifs montrent deux tendances envisageables d'évolution des besoins en eau aux horizons 2010 et 2020. Le scénario fondé sur l'hypothèse H1 (évolution linéaire) porte ainsi les besoins en eau à $720000 \mathrm{~m}^{3}$ en 2010 et $850000 \mathrm{~m}^{3}$ en 2020. La croissance des besoins est minorée selon le scénario fondé sur l'hypothèse $\mathrm{H} 2$ : ils atteignent respectivement $670000 \mathrm{~m}^{3}$ et $770000 \mathrm{~m}^{3}$ (fig. 2). Dans le cas où l'évolution des besoins en eau resterait assujettie au développement strictement résidentiel de l'île, il semble donc que la courbe de demande en eau s'infléchirait légèrement par rapport à la tendance de croissance linéaire des besoins observée entre 1975 et 2005.

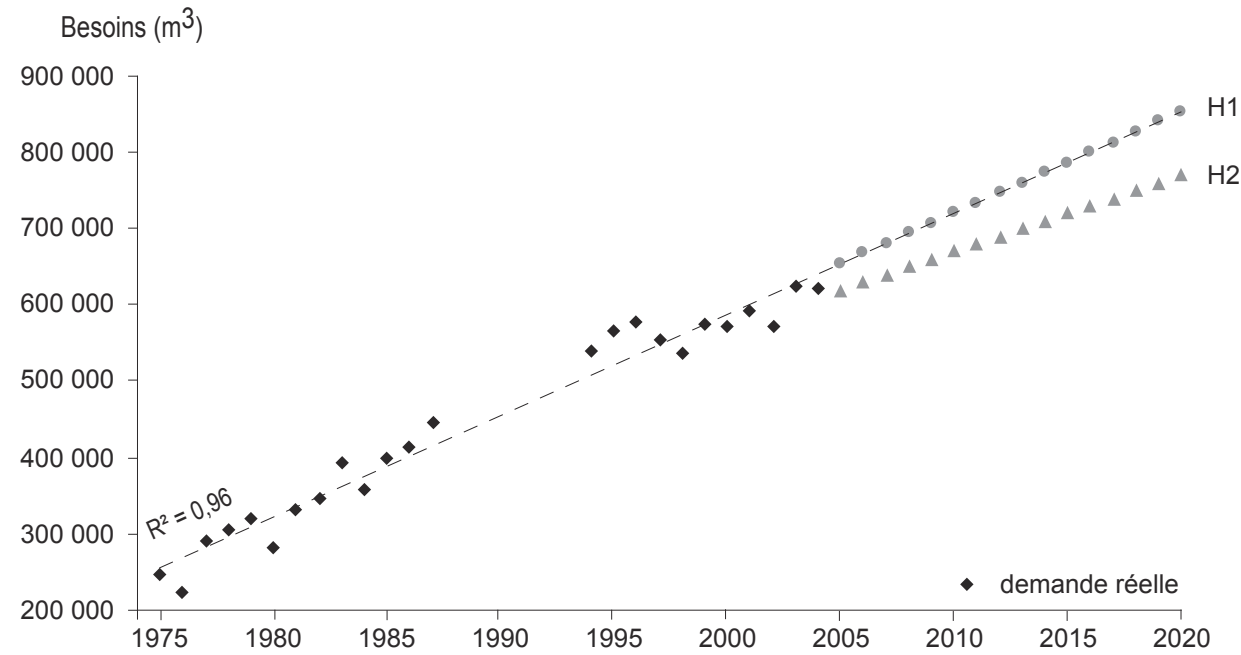

Figure 2 : Augmentation des besoins en eau à Belle-Île selon les scénarios $\mathrm{H} 1$ et $\mathrm{H} 2$ Water needs increase in Belle-île according to scenarios $\mathrm{H} 1$ and $\mathrm{H} 2$

Cependant, en conservant la situation hydraulique actuelle, l'autonomie de Belle-île diminue automatiquement pour n'être plus qu'annuelle en 2020 (tableau 5). Sans augmentation des surfaces réceptrices exploitées, le risque de non-satisfaction des besoins augmente inéluctablement : dans la situation la plus défavorable (H1), en 2020, les précipitations du premier semestre hydrologique ne seraient pas suffisantes une année sur vingt-cinq pour recharger les réserves hydriques à hauteur des besoins annuels. Les résultats soulignent ainsi la précarisation hydraulique de Belle-Île à court et surtout moyen terme si de nouvelles ressources hydriques - conventionnelles ou non - ne sont pas exploitées. 
QUEL RISQUE DE PÉNURIE D’EAU SUR LES ÎLES DU PONANT?

\begin{tabular}{|c|c|c|c|c|c|}
\hline Année & $\begin{array}{l}\text { Besoins annuels } \\
\qquad\left(\mathrm{m}^{3}\right)\end{array}$ & $\begin{array}{c}\text { Surfaces } \\
\text { réceptrices }\left(\mathrm{km}^{2}\right)\end{array}$ & $\begin{array}{c}\text { Réserves } \\
\text { hydriques }\left(\mathrm{m}^{3}\right)\end{array}$ & $\begin{array}{l}\text { Autonomie } \\
\text { hydraulique } \\
\text { (années) }\end{array}$ & $\begin{array}{l}\text { Fréquence } \\
\text { d'occurrence }\end{array}$ \\
\hline 1989 & 440000 & 7 & 330000 & 0,75 & $1 / 12$ \\
\hline 2005 & 600000 & 10 & 850000 & 1,4 & $1 / 13$ \\
\hline 2006 & 600000 & 24 & 850000 & 1,4 & $1 / 35$ \\
\hline \multirow{2}{*}{2010} & $(\mathrm{Hl}) 720000$ & \multirow{2}{*}{24} & \multirow{2}{*}{850000} & 1,2 & $1 / 30$ \\
\hline & $(\mathrm{H} 2) 670000$ & & & 1,25 & $1 / 32$ \\
\hline \multirow{2}{*}{2020} & $(\mathrm{Hl}) 850000$ & \multirow{2}{*}{24} & \multirow{2}{*}{850000} & 1 & $1 / 25$ \\
\hline & (H2) 770000 & & & 1,1 & $1 / 28$ \\
\hline
\end{tabular}

Tableau 5 : Évolution de la vulnérabilité hydraulique et du risque de pénurie d'eau à Belle-île Assessment of hydraulic vulnerability and water shortage risk in Belle-île

\section{L’enJeu CRUCial de la RÉCupération des EAUX DE PLUIE À MOLÈnE}

L'île de Molène a connu de nombreuses pénuries en eau au cours du XXe siècle. La dernière est celle de 1989 où elle a été ravitaillée par la Marine nationale dès le mois de novembre 1988 et au cours de l'été suivant. L'île n'avait à cette époque recours qu'à la seule récupération des eaux de pluie grâce à un impluvium de quelque $5000 \mathrm{~m}^{2}$; l'eau ainsi recueillie était stockée dans des citernes d'une capacité totale de $1850 \mathrm{~m}^{3}$. À la suite de la pénurie de 1989, des recherches d'eau souterraine ont permis d'exploiter le maigre aquifère de l'île, dont le volume exploitable moyen est estimé à $10000 \mathrm{~m}^{3}$. La capacité totale de stockage est alors portée à $11850 \mathrm{~m}^{3}$. L'optimisation de la ressource endogène a nettement réduit la vulnérabilité de Molène - celle-ci passant de 0,4 an en 1989 à près de deux ans en 2005 - et fait baisser le risque de rupture de l'alimentation en eau potable : il était annuel en 1989, il est presque dix fois moins grand en 2005 (tableau 6).

Par ailleurs, l'évolution interannuelle des besoins en eau ne laisse pas se dégager de tendance nette à une croissance linéaire comme dans le cas bellilois (fig. 3). Les variations d'une année à l'autre sont effectivement importantes : $7652 \mathrm{~m}^{3}$ distribués en 2003, $5992 \mathrm{~m}^{3}$ en 2004 par exemple. Les scénarios prospectifs fondés sur les hypothèses $\mathrm{H} 1$ et $\mathrm{H} 2$ montrent pourtant une évolution quasi similaire des besoins en eau à l'horizon 2020 : ceux-ci atteindraient respectivement $9900 \mathrm{~m}^{3}$ dans le cas d'une croissance linéaire des besoins et $9500 \mathrm{~m}^{3}$ dans la perspective de construction des trente-quatre nouvelles résidences inscrites au PLU (fig. 3). Ces besoins prévisionnels engendrent une diminution nette de l'autonomie hydraulique de l'île et augmentent inévitablement le risque que les volumes mobilisés lors de la recharge des réserves hydriques ne suffisent pas à les satisfaire. La pénurie serait ainsi à craindre pour tout déficit pluviométrique au premier semestre hydrologique caractérisé par une fréquence d'occurrence d'une année sur cinq (tableau 6).

Le défi molénais consiste à gérer la demande future sous l'effet d'une urbanisation certes modérée mais potentiellement menaçante compte tenu du contexte hydrologique de l'île. L'enjeu d'une gestion rationnelle des besoins en eau y est en effet d'autant plus crucial que l'ensemble des ressources hydriques est déjà exploité de façon optimale. Les résultats du scénario H2 bis montrent que l'équipement des nouvelles résidences en citernes de récupération de pluie aura un impact direct sur le risque de pénurie d'eau. Il limiterait l'inflation de la demande annuelle à $7400 \mathrm{~m}^{3}$ en 2020 : l'île conserverait ainsi une autonomie hydraulique légèrement supérieure à dix-huit mois et le risque de pénurie serait également atténué en comparaison des résultats issus des deux autres scénarios. Avec une fréquence d'occurrence d'une année sur huit, il n'en demeure pas moins élevé et place Molène dans une situation hydraulique relativement délicate. Bien que le maintien de l'usage des citernes de récupération des eaux de pluie soit inscrit au PLU à titre de 


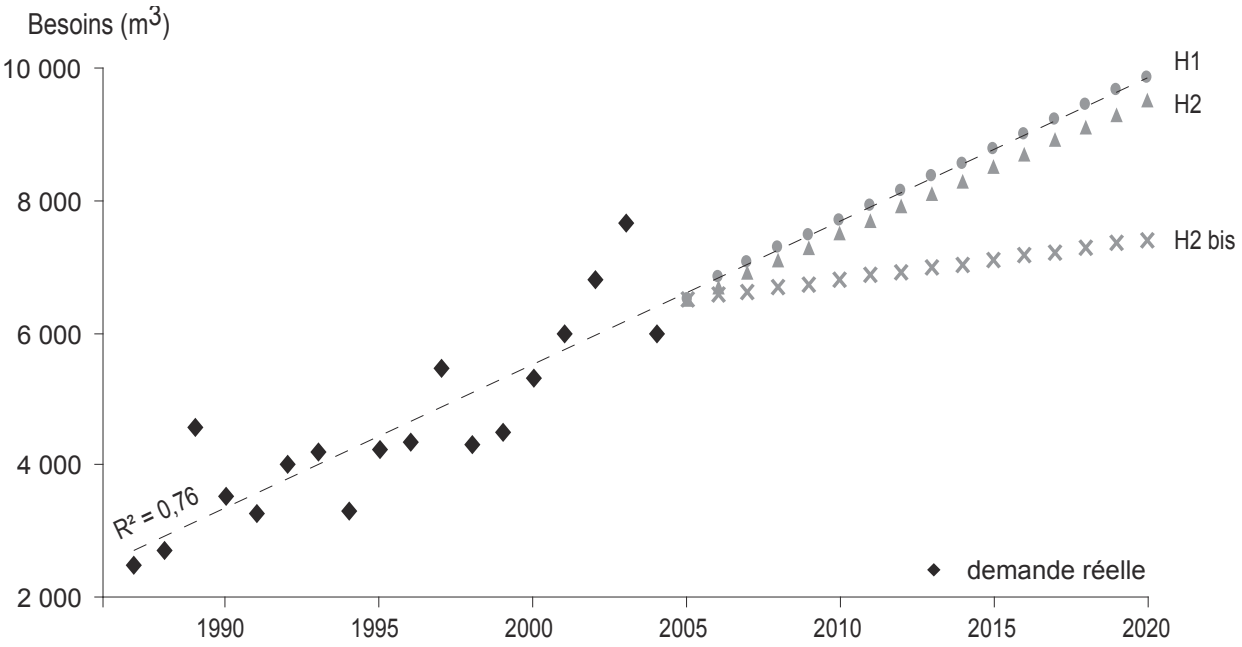

Figure 3 : Augmentation des besoins en eau à Molène, selon les scénarios $\mathrm{H} 1$, H2 et H2 bis Water needs increase in Molene according to scenarios $\mathrm{H} 1, \mathrm{H} 2$ and $\mathrm{H} 2$ bis

recommandation - « la construction d'une citerne personnelle est vivement recommandée de façon à gérer plus sûrement les réserves d'eau limitées de la commune », il est permis de s'inquiéter des conséquences urbanistiques sur la sécurisation de l'approvisionnement en eau potable des Molénais à moyen terme.

\begin{tabular}{|c|c|c|c|c|c|c|}
\hline Année & \multicolumn{2}{|c|}{$\begin{array}{l}\text { Besoins annuels } \\
\qquad\left(\mathrm{m}^{3}\right)\end{array}$} & $\begin{array}{c}\text { Surfaces } \\
\text { réceptrices (ha) }\end{array}$ & $\begin{array}{c}\text { Réserves } \\
\text { hydriques }\left(\mathrm{m}^{3}\right)\end{array}$ & $\begin{array}{c}\text { Autonomie } \\
\text { hydraulique } \\
\text { (années) }\end{array}$ & $\begin{array}{l}\text { Fréquence } \\
\text { d'occurrence }\end{array}$ \\
\hline 1989 & \multicolumn{2}{|c|}{4000} & 0,5 & 1850 & 0,4 & $1 / 1$ \\
\hline 2005 & \multicolumn{2}{|c|}{6500} & 10 & 11850 & 1,8 & $1 / 9$ \\
\hline \multirow{3}{*}{2010} & $(\mathrm{Hl})$ & 7700 & \multirow{3}{*}{10} & \multirow{3}{*}{11850} & 1,5 & $1 / 7$ \\
\hline & (H2) & 7500 & & & 1,6 & $1 / 7$ \\
\hline & (H2 bis) & 6800 & & & 1,7 & $1 / 9$ \\
\hline \multirow{3}{*}{2020} & $(\mathrm{Hl})$ & 9900 & \multirow{3}{*}{10} & \multirow{3}{*}{11850} & 1,2 & $1 / 5$ \\
\hline & (H2) & 9500 & & & 1,2 & $1 / 5$ \\
\hline & (H2 bis) & 7400 & & & 1,6 & $1 / 8$ \\
\hline
\end{tabular}

Tableau 6 : Évolution de la vulnérabilité hydraulique et du risque de pénurie d'eau à Molène Assessment of hydraulic vulnerability and water shortage risk in Molène

\section{Conclusion}

La démarche méthodologique présentée propose deux indicateurs complémentaires estimant la vulnérabilité hydraulique d'une part, quantifiant le risque de pénurie d'eau d'autre part. Ils donnent une définition statistique objective de ce dernier en agrégeant à la fois des paramètres structurels et sociaux (autonomie hydraulique), l'aléa climatique et des facteurs physiques et humains (risque de non-satisfaction des besoins). Ils peuvent ainsi aider à une gestion rationnelle du risque de rupture de l'alimentation en eau potable en testant différents scenarios prospectifs sur l'évolution des besoins mais aussi celle des infrastructures. Ils sont susceptibles de fournir aux différents 
gestionnaires des arguments de prise de décision en fonction de l'acceptabilité du risque. Cette première tentative de caractérisation du risque de pénurie devra être optimisée en intégrant la continuité interannuelle de la variation des réserves en eau : il s'agira de modéliser plus finement cette variation en prenant en compte le stock résiduel à la fin de l’année hydrologique, sa recharge partielle au cours du premier semestre hydrologique, et de la sorte ne plus se contenter d'une évaluation de non-satisfaction des besoins annuels.

Les résultats rappellent combien les îles ont été et continuent, à des degrés de risque désormais quantifiés et comparables, d'être exposées aux pénuries d'eau induites par la conjugaison des sécheresses, de leurs limites physiques et des pressions anthropiques sur leurs ressources hydriques. Les sécheresses ont d'ailleurs souvent été des événements révélateurs de ces limites naturelles et structurants dans les politiques d'équipement hydraulique des îles (recherche d'eaux souterraines après la sécheresse de 1989). Si la plupart d'entre elles ont choisi de se raccorder aux réseaux continentaux voire de dessaler l'eau de mer pour s'affranchir de leur insularité hydraulique, les six îles qui l'ont conservée doivent toujours gérer la contrainte climatique et la restriction naturelle de leurs ressources en eau. Les exemples de Belle-Île et Molène illustrent combien elles ne sont pas égales à ce sujet : la taille de Belle-Île offre évidemment un potentiel hydrologique superficiel intéressant, voire sécurisant, alors que l'exiguiité de Molène la place déjà dans une situation critique face à ses développements futurs. Les solutions envisagées pour sécuriser la production d'eau potable à moyen terme n'écartent pas totalement des raccordements aux réseaux continentaux, notamment dans le cas de Belle-Île. De tels transferts d'eau restent cependant très onéreux; dans le cas de petites îles, ils ne sont certainement pas compétitifs avec les technologies de dessalement de l'eau de mer.

En outre, la sécheresse n'est pas la seule menace climatique qui pèse sur les territoires insulaires : à plus long terme, les effets d'un changement climatique global qui semble aujourd'hui inéluctable risque de modifier les conditions hydroclimatiques des îles; de plus, la montée du niveau marin sera une source d'inquiétude supplémentaire compte tenu de ses conséquences sur les ressources souterraines insulaires. L'eau reste donc au cœur des enjeux des développements insulaires dont la durabilité dépend finalement de la résolution du dilemme entre limitation physique des ressources en eau et besoins anthropiques croissants.

\section{Bibliographie}

Anonyme, (2001). - Propositions de périmètres de protection pour les captages d'eaux communaux, rapport ANTEA à la commune de Molène, 15 p.

Bioret (F.), Brigand (L.), Le Démézet (M.), 1991. - « L'environnement : argument de développement d'un tourisme intégré dans les îles bretonnes ", dans Territoires et sociétés insulaires, Paris, ministère de l'Environnement, coll. « Recherches Environnement », n 36, p. 399-406.

Brigand (L.), 1983. - Les îles de Bretagne. Aspects géographiques de l'insularité, thèse de doctorat, Université de Bretagne Occidentale, 2 tomes, 267 p. et 111.

Brigand (L.), 1999. - « Les enjeux touristiques dans les îles du Ponant », Islands 2000 ; the world of islands : what development of the eve of the year 2000?, actes de la conférence internationale de Giardiny-Naxos, Italie, 1992, NTUA, UNESCO, INSULA, p. 51-61.

Bunot (C.), 2006. - Marché du logement et division sociale de l'espace dans les îles du Ponant, thèse de doctorat, Université de Bretagne Occidentale, 445 p.

Castany (G.), 1982. - Principes et méthodes de l'hydrogéologie, Paris, Dunod Université/Bordas, 236 p.

Chiron (T.), Dubreuil (V.), 2006. - «Sécheresses et risque de pénurie d'eau sur les petites îles côtières françaises », dans Les risques liés au temps et au climat, actes du $19^{\mathrm{e}}$ colloque de l'Association Internationale de Climatologie, 6-8 septembre 2006, Épernay, France, p. 160-165.

DAuPHINÉ (A.), 2003. - Risques et catastrophes. Observer, spatialiser, comprendre, gérer, Paris, Armand Colin, 288 p. 
Direction Départementale de l’Agriculture et de la Forêt du Morbihan, 1991. - Île d'Hoëdic, compte rendu des travaux de recherches d'eaux souterraines, $4 \mathrm{p}$.

Dubreuil (V.), 1994. - La sécheresse dans la France de l'Ouest. Étude d'après les bilans hydriques et les données des satellites NOAA-AVHRR, thèse de doctorat, Université de Rennes 2, 2 tomes, 381 p. et 550 p.

Dumortier (B.), 1976. - Belle-Île, Houat, Hoëdic. Le poids de l'insularité dans trois îles de Bretagne méridionale, École Normale Supérieure de Jeunes Filles, Paris, 163 p.

Guilcher (A.), 1977. - «Vie et mort des petites îles finistériennes », Norois, n 95, p. 123-128.

Kahn (H.), Wiener (A.-J.), 1968. - L'an 2000, Paris, Robert Laffont, 521 p.

Marchand (J.-P.), 1981. - «La variabilité de l'organisation mensuelle des précipitations. L'exemple de Dublin Phoenix Park (1838-1976) », dans PAgney (P.) (dir.), Eaux et climats, Mélanges géographiques offerts en hommage à Charles-Pierre Péguy, Grenoble, CNRS, p. 325-337.

Margat (J.), 2005. - «Quels indicateurs pertinents de la pénurie d'eau? », Géocarrefour, vol. 80, n 4, p. 261-262.

Mounier (J.), 1980. - «Fluctuations climatiques récentes en Bretagne », Norois, n 107, p. 460-463.

Patturel (L.), 1972. - « La station de dessalement d'eau de mer de Houat (Morbihan) », Le Moniteur des travaux publics et du bâtiment, $\mathrm{n}^{\circ} 29$, p. 151-156.

Peguy (C.-P.), Mounier (J.), Charre (J.), Douguedroit (A.), Delannoy (H.), Marchand (J.-P.), Vivian (H.), 1981. - « Dix ans de carte climatique détaillée de la France au 1/250 000 », dans PAGNey (P.) (dir.), Eaux et climats, Mélanges géographiques offerts en hommage à Charles-Pierre Péguy, Grenoble, CNRS, p. 41-84.

Péron (F.), 1993. - Des îles et des hommes. L'insularité aujourd'hui, Rennes, Éditions de la Cité/OuestFrance, $287 \mathrm{p}$.

Poux (X.), 2005. - « Fonctions, construction et évaluation des scénarios prospectifs », dans Mermet (L.) (dir.), Étudier des écologies futures, Bruxelles, PIE-Peter Lang, coll. « Ecopolis », p. 151-186.

Rivière-Honegger (A.), Bravard (J.-P.), 2005. - « La pénurie d'eau : donnée naturelle ou question sociale? », Géocarrefour, vol. 80, n 4, p. 257-260.

Singelin (J.-Y.), Monnat (P.), 1991. - « Gestion des ressources et aménagement des îles (rapport sur le troisième thème) ", dans Territoires et sociétés insulaires, Paris, ministère de l'Environnement, collection "Recherches Environnement », n³ 36, p. 433-435.

Sogreah-Praud, 2004. - Étude hydrogéologique et modélisation de lîle d'Houat, rapport pour le Conseil Général et la direction départementale de l'Agriculture et de la Forêt du Morbihan, 120 p.

Viers (G.), Vigneau (J.-P.), 1990. - Éléments de climatologie, Paris, Nathan Université, 223 p.

Cet article a été reçu le 10 octobre 2006 et définitivement accepté le 12 mars 2007. 